Research Paper

\title{
Effects of Long Form of CAPON Overexpression on Glioma Cell Proliferation are Dependent on AKT/mTOR/P53 Signaling
}

Dong Liang1,2*, Yunnong Song $12^{*}$, Guangwei Fan ${ }^{1,2^{*}}$, Daofei Ji3 Liang ${ }^{1,2}$, Rutong $\mathrm{Yu}^{1,2}{ }^{\bowtie}$, Shangfeng Gao ${ }^{1,2}$

1. Institute of Nervous System Diseases, Xuzhou Medical University, 84 West Huai-Hai Road, Xuzhou 221002, Jiangsu, China;

2. Department of Neurosurgery, The Affiliated Hospital of Xuzhou Medical University, 99 West Huai-Hai Road, Xuzhou 221002, Jiangsu, China;

3. Department of Neurosurgery, The Second Hospital of Xuzhou Medical University, 32 Mei-Jian Road, Xuzhou 221006, Jiangsu, China.

*These authors contributed equally to this work.

$\square$ Corresponding authors: Shangfeng Gao and Rutong Yu, Institute of Nervous System Diseases, Xuzhou Medical University, 84 West Huai-Hai Road; Department of Neurosurgery, The Affiliated Hospital of Xuzhou Medical University, 99 West Huai-Hai Road, Xuzhou 221002, Jiangsu, China. Emails: gaoshangfeng@xzhmu.edu.cn (S.G.); yu.rutong@163.com (R.Y.)

(c) Ivyspring International Publisher. This is an open access article distributed under the terms of the Creative Commons Attribution (CC BY-NC) license (https://creativecommons.org/licenses/by-nc/4.0/). See http://ivyspring.com/terms for full terms and conditions.

Received: 2018.11.16; Accepted: 2019.03.21; Published: 2019.04.25

\begin{abstract}
Background: CAPON has two isoforms in human brain: long form of CAPON (CAPON-L) and short form of CAPON (CAPON-S). Recent studies have indicated the involvement of CAPON in tumor cell growth. We aimed to reveal the role of the two CAPON isoforms in the proliferation of glioma cells in this study.

Materials and Methods: Lentivirus-mediated stable cell lines with CAPON-L or CAPON-S overexpression were established in U87 and U251 glioma cells. Cell counting kit-8 and colony formation assays were used to evaluate cell proliferation. Western blot analysis of cell cycle-related proteins and flow cytometry were performed to analyze cell cycle progression. Some important molecules of the AKT/mTOR pathway and P53 were also measured by Western blot analysis.

Results: Overexpression of CAPON-L showed a significantly inhibitory role in U251 cells, while it exhibited a promoting role in U87 cells. Consistently, overexpressing CAPON-L impeded the cell cycle progression and down-regulated the expression levels of Cyclin D1, CDK4 and CDK6 in U251 cells, whereas it up-regulated the CDK6 level in U87 cells. The overexpression of CAPON-L significantly decreased the phosphorylation and/or total levels of AKT, mTOR and S6 in U251 cells, while it did not affect these signaling molecules in U87 cells, except for a significant increase in the phosphorylation of AKT at Thr-308 site. Transfecting constitutively active AKT (myr-AKT) partially reversed the decreased phosphorylation of AKT and S6 in the CAPON-L-overexpressing U251 cells. In addition, we found a significant decrease in the wild-type P53 level in the CAPON-L-overexpressing U87 cells. The overexpression of CAPON-S also inhibited cell proliferation, blocked cell cycle progression, and decreased the AKT/mTOR pathway activity in U251 cells.

Conclusion: The effects of CAPON-L overexpression on glioma cell proliferation are dependent on the AKT/mTOR/P53 activity. The overexpression of CAPON inhibits U251 cell proliferation through the AKT/mTOR signaling pathway, while overexpressing CAPON-L promoted U87 cell proliferation, possibly through down-regulating the P53 level.
\end{abstract}

Key words: NOS1AP, U87, U251, cell cycle

\section{Introduction}

Glioma is the most common malignant tumor in the central nervous system [1]. Although advances have been made using multimodal therapy regimens, such as surgical operation, radiotherapy and chemotherapy, patients with malignant gliomas have experienced little change in survival time [2]. The 5 -year survival is below $10 \%$ and the average time from diagnosis to death is less than one and a half years [3]. The difficulties in curing glioma are due to uncontrolled proliferation and infiltrative growth [4]. 
Therefore, it is urgently needed to search for effective therapeutic targets, particularly those related to glioma cell proliferation.

CAPON (Carboxy-terminal PDZ ligand of NOS1) was first identified in the rat brain, it is also known as a nitric oxide synthase 1 (NOS1) adaptor protein (NOS1AP) [5]. CAPON is widely expressed in a variety of tissues including the brain, cardiac muscle [6], skeletal muscle [7], and pancreas [8]. CAPON has at least two isoforms in human brain: long form of CAPON (CAPON-L) and short form of CAPON (CAPON-S) [9]. CAPON-L consists of a phosphotyrosine-binding (PTB) domain, a PSD-95/discs-large /ZO-1 (PDZ)-binding motif, and a middle region between them; CAPON-S is a truncated form of CAPON-L, only containing the PDZ-binding motif [10]. CAPON plays a significant role in the pathophysiology of some neurological and psychiatric disorders [10].

CAPON, as an interacting protein of NOS1, is capable of regulating nitric oxide (NO) production in the brain [5], and NO is known for its crucial role in angiogenesis and tumor cell survival [11]. CAPON binds to Dexras1 [12], a member of RAS superfamily. Analysis of the processing and signaling mechanisms of the RAS superfamily has identified potential targets for tumor treatment [13]. More importantly, CAPON links with scribble and scribble in turn binds to Yes-associated protein (YAP), thus forming a ternary complex [14]. Both scribble and YAP are involved in tumor cell proliferation and migration [15, 16]. These studies suggested the possible involvement of CAPON in tumor cell growth and expansion.

Emerging data have showed an inhibitory role of CAPON in tumor cell growth. Overexpressing CAPON inhibited breast cancer cell proliferation [14, 17]. Silencing CAPON enhanced anchorage- independent growth in breast cancer cells [18]. In addition, interference with CAPON expression promoted the proliferation of multiple myeloma cells [19]. We previously reported that overexpression of CAPON-S inhibited cell proliferation and decreased the activity of AKT signaling in U87 cells [20]. However, it is largely unknown about the role of CAPON-L in glioma cell proliferation.

\section{Materials and Methods}

\section{Cell culture}

HEK293T cells and glioma cell lines U87 and U251 were purchased from Shanghai Cell Bank, Type Culture Collection Committee, Chinese Academy of Sciences. The cells were grown in DMEM (293T, U251) or MEM (U87) supplemented with $10 \%$ fetal bovine serum (FBS, Gibco). All cell lines were cultured in a cell incubator containing $5 \% \mathrm{CO}_{2}$ under the saturated humidity at $37^{\circ} \mathrm{C}$.

\section{Antibodies}

Rabbit anti-CAPON antibody was purchased from Santa Cruz Bio. (1:500, Santa Cruz, CA, USA) and could react with both CAPON-L and CAPON-S [21]. Antibodies against GFP (1:5000, Abbkine, Redlands, CA, USA), Cyclin D1, CDK4, CDK6, AKT, p-AKT (Thr308 and Ser473), S6, p-S6 (Ser235/236), mTOR, p-mTOR (Ser2448) and P53 (1:1000, Cell Signaling Technology, Denver, CO, USA) are commercially available.

\section{Lentivirus construction, production, and infection}

To construct CAPON-L lentivirus plasmid, the human capon variant 1 (Accession number: NM_014 697) was inserted into the pCDH-GFP-puro plasmid at Nhe I and Not I sites. To construct the CAPON-S lentivirus plasmid, the capon variant 2 was inserted into the pWPXLd-puro plasmid at BamH I and Mlu I. The CAPON-L was expressed independently, while the CAPON-S was expressed together with GFP protein. The viruses were propagated in 293T cells and used to infect glioma cells according to the literature [20]. In $48 \mathrm{~h}$ after infection, the virusinfected cells were cultured in the medium containing $2.5 \mu \mathrm{g} / \mathrm{mL}$ puromycin for selection. The surviving cells were used in the subsequent experiments.

\section{Cell viability detection}

A Cell Counting Kit (CCK-8, Dojindo, Tokyo, Japan) was used to assess cell viability. The single cell suspension $\left(2 \times 10^{3}, 100 \mu \mathrm{L}\right)$ were dispensed in a 96-well plate and cultured for 6, 24, 48, 72, and $96 \mathrm{~h}$, respectively. Then, $10 \mu \mathrm{L}$ of the CCK- 8 reagent was added into each well and incubated for another $1 \mathrm{~h}$. The absorbance value at $450 \mathrm{~nm}$ was measured using a scanning microplate reader. Cell viability at individual time point was normalized to the absorbance value at $6 \mathrm{~h}$ for each group.

\section{Colony formation assay}

Colony formation assay was performed using the same protocol as described in our previous publication [22]. Briefly, four hundred cells were seeded in a 6-well plate and cultured for 2-3 weeks. The cells were fixed and then stained with $0.05 \%$ crystal violet. The plates were photographed with a camera. Colonies containing more than 50 cells were counted.

\section{Flow cytometry}

The cell cycle was assessed by flow cytometry using a commercial cell cycle analysis kit (NewMed 
Cytomics, Suzhou, China). According to the manufacturer's protocol, cells were trypsinized into single-cell suspension and collected by centrifugation at $1500 \mathrm{rpm}$. The reagents $\mathrm{A}, \mathrm{B}$, and $\mathrm{C}$ from the kit were successively added into the cells. The cell suspension was filtered and immediately analyzed by flow cytometry (BD, Franklin Lakes, NJ, USA).

\section{Western blot analysis}

Total protein was extracted from the cultured cells according to a previously described method [23]. Protein concentrations were determined by a BCA Protein Assay Kit (Beyotime, Haimen, China). Equal amount of total protein was used for Western blot with a similar protocol as reported earlier this year [24]. $\beta$-Actin (1:1500, Santa Cruz Bio.) was used as a protein-loading control. Band densities were analyzed using Image J software (National Institute of Health, Bethesda, MD, USA). The relative protein levels were determined by normalizing the densitometry value of proteins of interest to that of $\beta$-Actin.

\section{Statistical analysis}

Quantitative data were obtained from at least three independent experiments and expressed as mean \pm S.E.M. Comparison between two groups was analyzed by unpaired Student's $t$ test. Statistical analyses were performed using SPSS version 13.0 (SPSS Inc., Chicago, IL, USA). Tests were two-tailed and values of $P<0.05$ were considered to be significant.

\section{Results}

\section{Efficiency of CAPON-L overexpression in glioma cells}

We established stable glioma cell lines with overexpression of CAPON-L in U87 and U251 cells by lentivirus infection. Fluorescence microscopy observation showed that $80 \%$ of lentivirus-infected cells had GFP fluorescence (Figure 1A). Western blot analysis using the CAPON antibody further confirmed that the CAPON-L was abundantly overexpressed both in U87 and in U251 cells (Figure 1B). These data indicated that the lentivirus-mediated stable cell lines with CAPON-L overexpression were successfully established in glioma cells.

\section{Effects of CAPON-L overexpression on the proliferation of glioma cells}

CCK8 assay showed that overexpression of CAPON-L increased the cell viability at $48 \mathrm{~h}(P=$ $0.032), 72 \mathrm{~h}(P=0.029)$ and $96 \mathrm{~h}(P=0.003)$ in U87 cells, while overexpressing CAPON-L significantly decreased the cell viability at $48 \mathrm{~h}(P=0.001), 72 \mathrm{~h}(P<$ $0.001)$ and $96 \mathrm{~h}(P=0.001)$ in U251 cells (Figure $2 \mathrm{~A})$.
Similarly, colony formation assays revealed an increase in the number of colonies in CAPON-Loverexpressing U87 cells $(P=0.108)$ and a reduction in the number of colonies in CAPON-Loverexpressing U251 cells $(P=0.078)$ (Figure $2 \mathrm{~B}, \mathrm{C})$. These results indicated that the overexpression of CAPON-L promoted the proliferation in U87 cells and inhibited the proliferation in U251 cells.

A
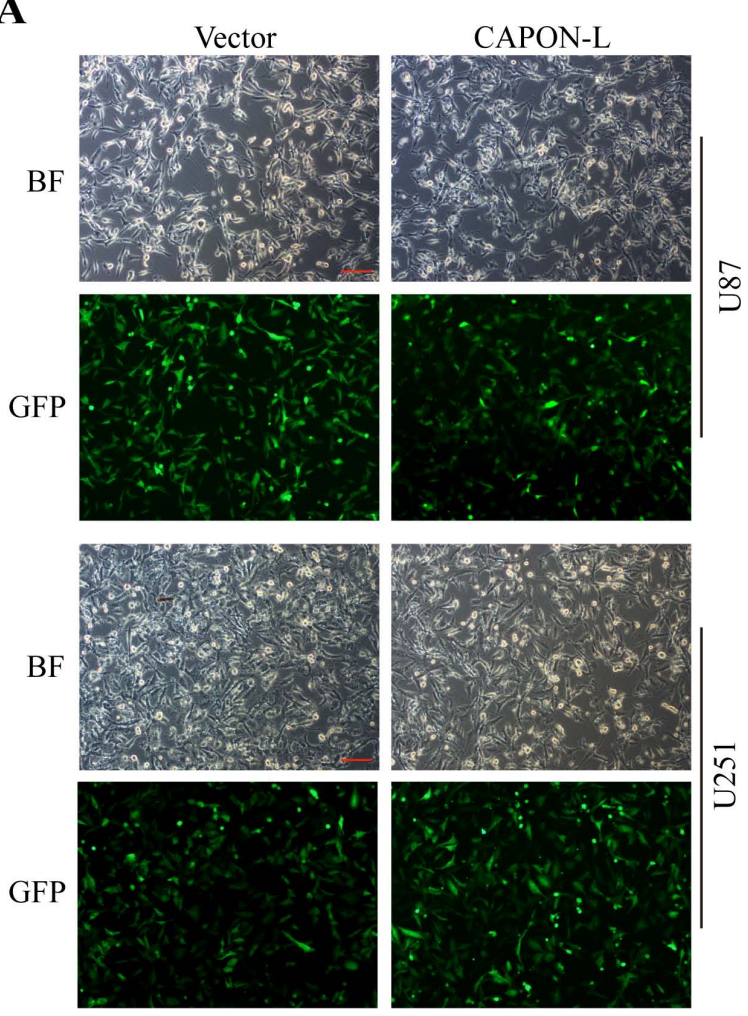

B

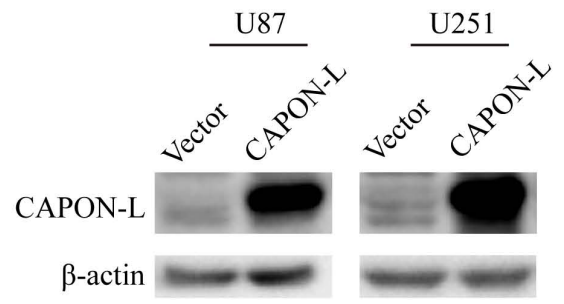

Figure 1. Identification of the efficiency of CAPON-L overexpression in glioma cells. (A) Lentivirus infection efficiency was indicated by bright field (BF) and GFP fluorescence in Vector group and CAPON-L group. Approximately $80 \%$ of U87 and U251 cells were infected by the lentivirus from Vector group and CAPON-L group. Scale bars: $200 \mu \mathrm{m}$. (B) Western blot showed that CAPON-L was abundantly overexpressed in the CAPON-L group both in U87 and U251 cells.

\section{Effects of CAPON-L overexpression on the cell cycle progression of glioma cells}

Flow cytometry showed that overexpressing CAPON-L showed no significant changes in the cell distribution in the G0/G1 or $S$ phase, and an increase for the percentage of cells in G2/M phase in U87 cells 
$(P=0.137)$ (Figure 3A, B). In U251 cells, however, the overexpression of CAPON-L arrested the cells in the G0/G1 phase $(P=0.001)$ and reduced the percentage of cells in the $S(P=0.109)$ and $\mathrm{G} 2 / \mathrm{M}$ phases $(P=$ 0.003 ) (Figure 3A, B). We further measured the changes of cell cycle-related proteins. The protein levels of Cyclin D1 $(P=0.006)$ and Cyclin-Dependent Kinases CDK4 $(P=0.024)$ and CDK6 $(P<0.001)$ were significantly decreased in the CAPON-L-overexpressing U251 cells (Figure 4A, B), while they were increased in the CAPON-L-overexpressing U87 cells, and CDK6 reached a statistically significant increase

A
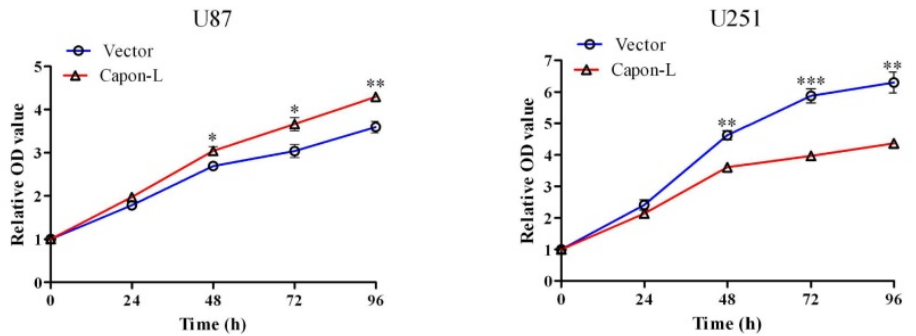

B
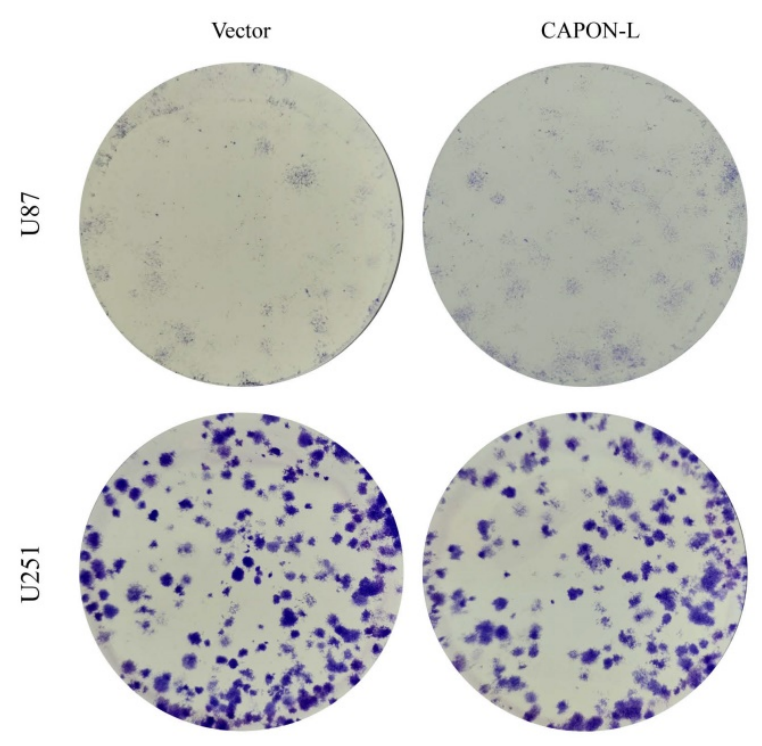

C
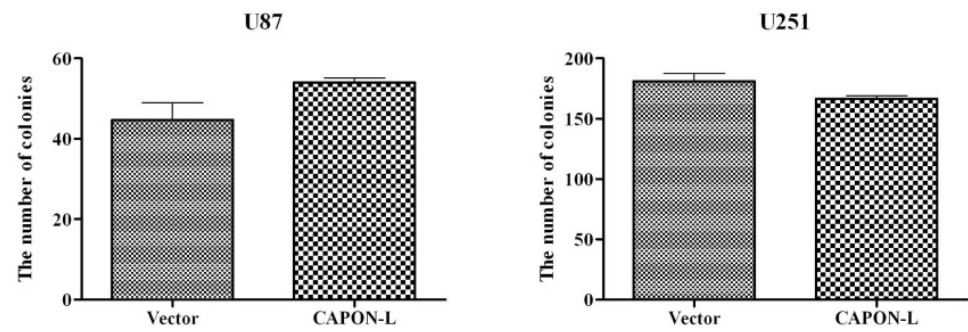

Figure 2. Effects of CAPON-L overexpression on the proliferation of glioma cells. (A) CCK8 assay was used to measure the cell viability in CAPON-L-overexpressing U87 and U251 cells. The overexpression of CAPON-L caused an increase in U87 cells and a significant decrease in U251 cells in the cell viability at the indicated time. (B, C) Colony formation assay was used to evaluate the proliferation in CAPON-L-overexpressing U87 and U251 cells. Representative images for the plate colony are shown in B. Quantification for the number of colonies revealed an increase in the CAPON-L- overexpressing U87 cells and a reduction in the CAPON-L-overexpressing U 251 cells (C). $(* P<0.05 ; * * P<0.01 ; * * * P<0.001)$.
$(P=0.002)$ (Figure 4A, B). These data suggested that the overexpression of CAPON-L significantly impeded the cell cycle progression in U251 cells, but not in U87 cells.

\section{Effects of CAPON-L overexpression on the AKT/mTOR/P53 signaling}

We previously found by antibody array in U87 cells that overexpression of CAPON-S reduced the phosphorylation of AKT, mTOR and S6 [20]. Here, we assessed the activity of AKT/mTOR signaling by Western blot in the CAPON- L-overexpressing glioma cells. In U251 cells, the overexpression of CAPON-L caused significant decreases in the p-AKT $(473)(P=0.029)$, p-mTOR $(P=$ $0.030)$, mTOR $(P=0.008), \mathrm{p}-\mathrm{S6}(P<0.001)$ and S6 $(P=0.047)$ levels, and a non-significant decrease in the $\mathrm{p}$-AKT (308) $(P=0.074)$ level (Figure 5A, B); In U87 cells, however, overexpressing CAPON-L showed no significant effects on these signaling molecules except a significant increase in the p-AKT (308) level $(P<0.001)$ (Figure 5A, B). Importantly, transfecting the constitutive active AKT (myr-AKT) plasmid into the CAPON-L-overexpressing U251 cells partially reversed the decreased phosphorylation of AKT and S6 caused by overexpression of CAPON-L (Figure 5C). In addition, we found an increase in the muted P53 level in the CAPON-L-overexpressing U251 cells ( $P=$ $0.095)$ and a significant decrease in the wild-type P53 level in U87 cells $(P=0.002)$ (Figure 5D). It should be noted that the wild-type P53 was becoming detectable in U87 cells under an overexposure condition (30 min).

\section{Effects of CAPON-S overexpression on the cell proliferation, cell cycle progression and AKT/mTOR signaling in glioma cells}

The same CAPON-S lentivirus plasmid that we constructed before [20] was used to establish stably up-regulated cell lines in U251 cells. The infection and overexpression efficiency were evaluated by fluorescence microscopy and Western blot, respectively (Figure S1). In CAPON-S-overexpressing U251 cells, CCK8 assay showed that cell viability was decreased at $24 \mathrm{~h}(P=0.018), 48 \mathrm{~h}(P=$ $0.059), 72 \mathrm{~h}(P=0.001)$ and $96 \mathrm{~h}(P=0.001)$ 
(Figure 6A), colony formation assay revealed a significant decrease in the colony numbers $(P=0.001$, Figure 6B), and flow cytometry exhibited cell cycle arrest in the G0/G1 phase $(P=0.001)$ and a reduced cell distribution in the $S$ phase $(P=0.003)$ (Figure 6C). In addition, the overexpression of CAPON-S significantly decreased the AKT/mTOR pathway activity as reflected by the reduced levels for the
p-AKT $(473)(P=0.044)$, p-AKT $(308)(P=0.065)$, AKT $(P=0.066)$, p-S6 $(P=0.032)$ and S6 $(P=0.035)$ in U251 cells (Figure 6D, E). Together with our previous findings in U87 cells [20], these data suggested that the overexpression of CAPON-S significantly inhibited the cell proliferation and blocked the cell cycle progression in both U87 and U251 cells.
A U87
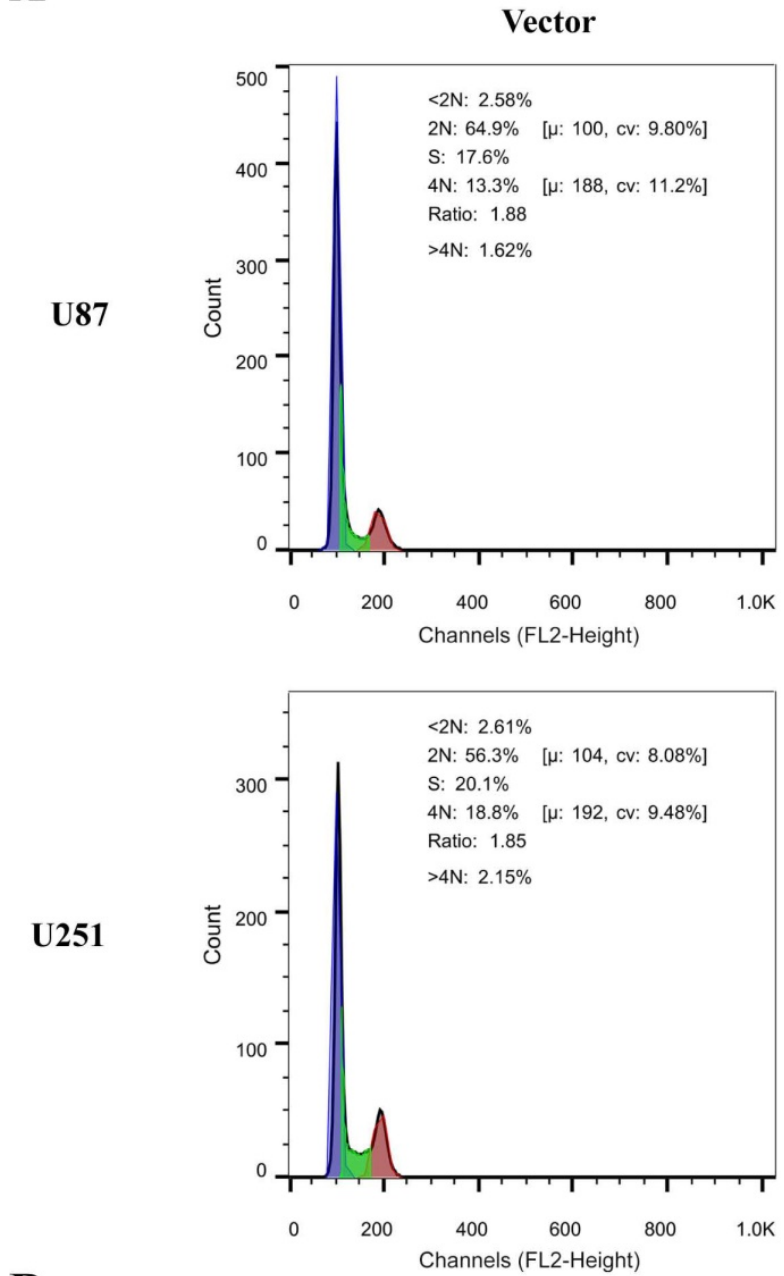

U87

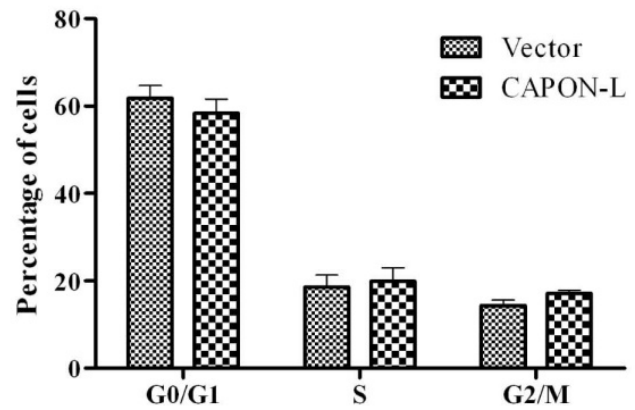

CAPON-L
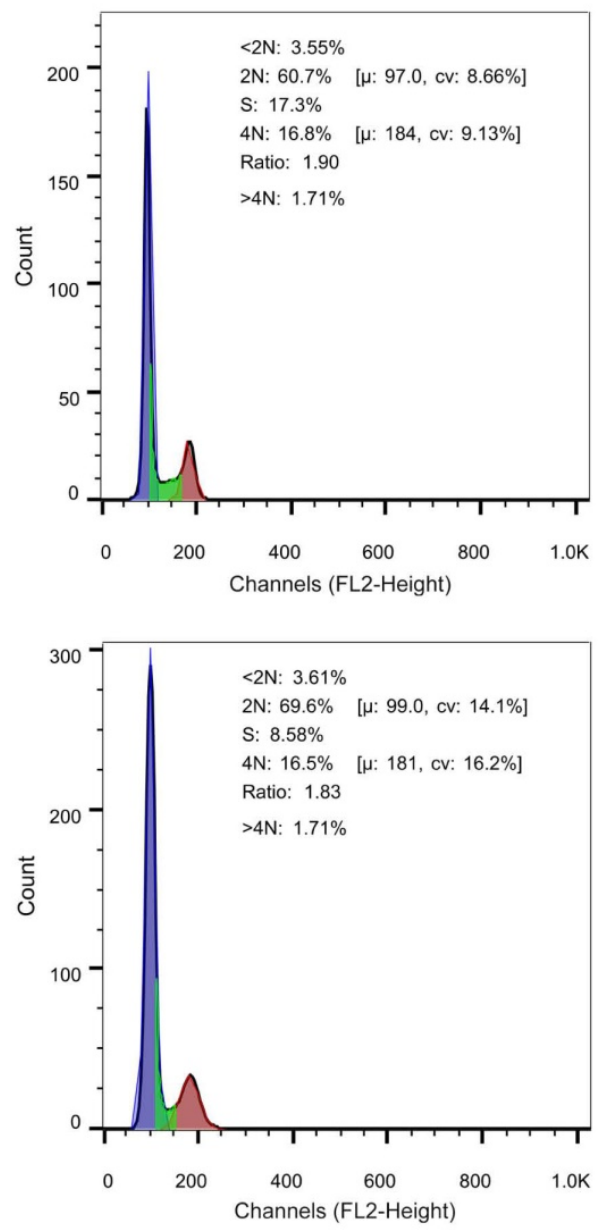

$\mathbf{U} 251$

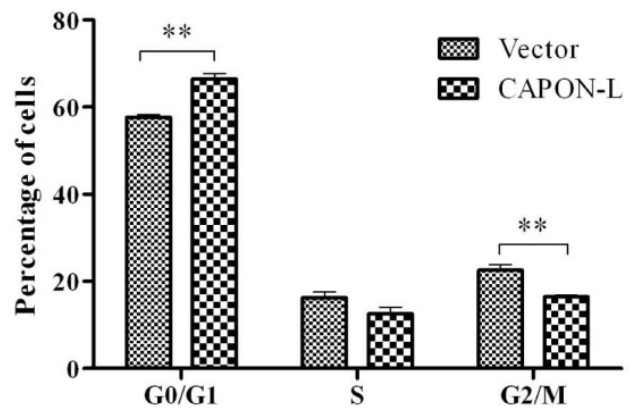

Figure 3. Effects of CAPON-L overexpression on the cell cycle progression in glioma cells. Flow cytometry was employed to analyze the cell cycle progression in CAPON-L-overexpressing glioma cells. Representative histograms for U87 and U251 cells are shown in A. Quantification graphs showed that the overexpression of CAPON-L showed no significant changes in the G0/GI, S or G2/M phase in U87 cells, while it significantly decreased the percentage of cells in the S phase and arrested the cells in the G1 phase in U251 cells $(\mathbf{B}) .(* * P<0.01)$. 


\section{Discussion}

We previously reported that overexpression of CAPON-S inhibited cell proliferation and impeded cell cycle progression in U87 cells [20]. In this study, we obtained the similar results for CAPON-S in U251 cells. However, stably overexpressing CAPON-L showed different effects on cell proliferation in U87 and U251 cells, that is, a promoting role in U87 cells versus a significantly inhibitory role in U251 cells. More signaling molecules in the AKT/mTOR and P53 pathways were significantly altered in U251 cells than in U87, which might be responsible for the different effects as we observed in CAPON-L-overexpressing U251 and U87 glioma cell lines. In addition, overexpression of myr-AKT rescued the decreased AKT pathway activity caused by overexpression of CAPON-L in U251 cells.

We found that overexpressing CAPON led to cell growth inhibition and cell cycle arrest in U251 cells. This was consistent with the findings in breast cancer cells $[14,18]$. Down-regulation of CAPON enhanced anchorage-independent cell growth, whereas overexpression of CAPON inhibited cell growth [14, 18]. Interestingly, there was a big difference between CAPON-L and CAPON-S in their effects on cell proliferation and cell cycle progression

A
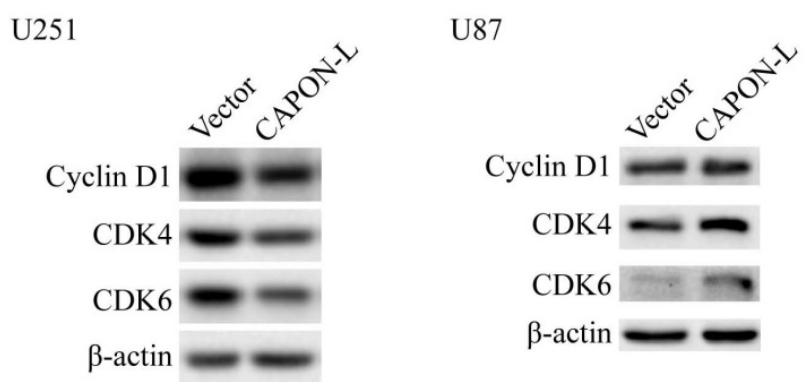

B
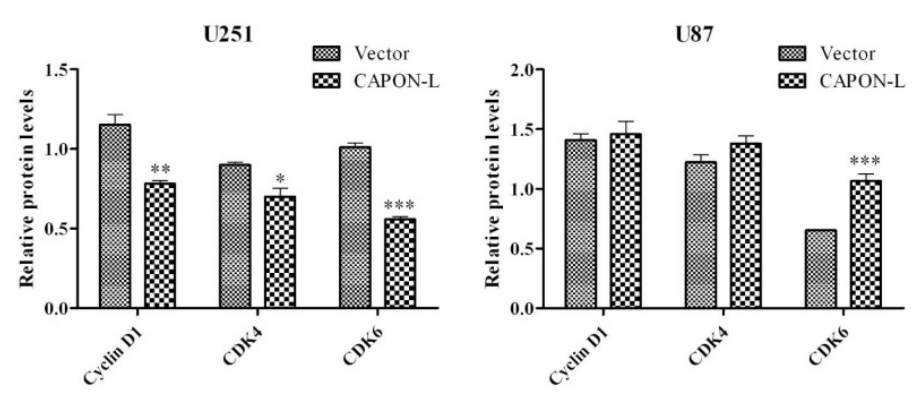

Figure 4. Effects of CAPON-L overexpression on the expression of cell cycle-related proteins. Western blot was used to measure the changes of cell cycle-related proteins in CAPON-L-overexpressing glioma cells. Representative blot images for U251 and U87cells are shown in A. Quantification graphs showed that the overexpression of CAPON-L caused significant decreases in the Cyclin D1, CDK4 and CDK6 protein levels in $\mathrm{U} 251$ cells, and that it significantly up-regulated the CDK6 level but not changed the levels of CDK4 and CDK6 (B). ( $* P<0.05$; ** $P<0.01$; *** $P<0.001)$. in U87 cells. Overexpressing CAPON-L showed a promoting role in cell proliferation, in contrast to the inhibitory effects of CAPON-S overexpression on cell proliferation and cell cycle progression. Structurally, both CAPON-L and CAPON-S have the PDZ-binding motif, but CAPON-S is a truncated version of CAPON-L, lacking the PTB domain and the middle region that CAPON-L have [10]. Therefore, we speculated that the PTB or the middle region might lead to the different role in cell proliferation between CAPON-L and CAPON-S in U87 cells, which deserves further investigations.

Based on our previous antibody array data that overexpression of CAPON-S decreased the activity of AKT signaling in U87 cells [20], we detected some important signaling molecules of this pathway in the CAPON-L-overexpressing glioma cells. Overexpression of CAPON-L decreased the phosphorylation of AKT at both 473 and 308 sites in U251 cells. However, in U87 cells, only the p-AKT (308) level showed a significant increase by CAPON-L overexpression. Since AKT mediates negative control of p53 levels through enhancing MDM2-mediated targeting of p53 for degradation [25], we then detected the P53 protein level. In U87 cells, the P53 was wild-type and hard to detect, but overexpressing CAPON-L caused a significant decrease in the P53 level at a longer exposure time (30 $\mathrm{min})$. The increased p-AKT (308) and the decreased P53 levels may account for the promoting role of CAPON-L in U87 cell proliferation. In U251 cells, the overexpression of CAPON-L showed no significant changes in the muted P53 level, which does not appear to be responsible for its significant inhibition of cell proliferation. Interestingly, the phosphorylation and total levels of both mTOR and S6 were significantly decreased in the CAPON-L-overexpressing U251 cells, while they were unchanged in the CAPON-L- overexpressing U87 cells. Thus, we proposed that the AKT-mTOR-S6 pathway may be responsible for the inhibitory effects of CAPON-L overexpression on cell proliferation in U251 cells. This was further supported by the rescue experiment using a constitutively active AKT (myr-AKT) plasmid.

Flow cytometry showed that the overexpression of CAPON- L led to more cells in the G0/G1 phase and less cells in S phase in U251 cells, indicating the cell cycle arrest. This was further supported by the decreased levels of cell cycle-related proteins (Cyclin D1, CDK2 and CDK6) that were known to be regulated by the activity of AKT [26]. Therefore, the overexpression of CAPON-L inhibited cell prolifera- 
tion, possibly through AKT-mediated cell cycle arrest in U251 cells. Moreover, overexpressing CAPON-L significantly decreased the activity and expression level of mTOR and S6, both of which are required for ribosome and protein synthesis [27]. Thus, CAPON-L may also play an inhibitory role in cell proliferation through restricting global protein synthesis. All these possibilities warrant further studies.

A few limitations should be mentioned here. Firstly, we found in U251 cells CAPON-L played an inhibitory role in glioma cell proliferation, other

A

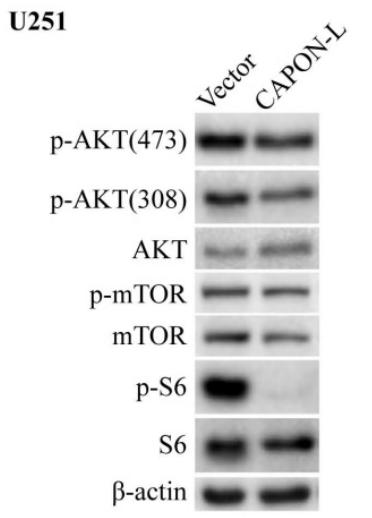

B

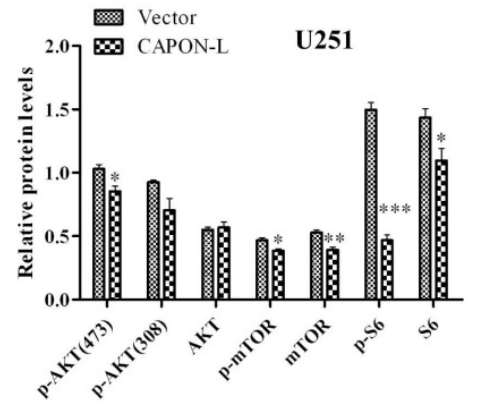

C

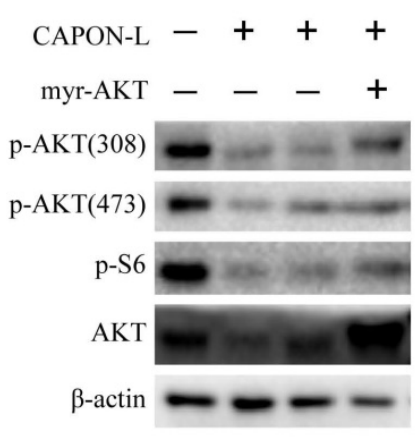

human cell lines with P53 mutant or depletion are needed to confirm this conclusion. Secondly, we previously silenced CAPON in rat $\mathrm{C} 6$ cells and found a promoting effect on glioma cell proliferation. However, in human glioma cell lines, for example, U251 and U87, the expression of CAPON-L is very low, we therefore did not confirm our results in CAPON-down-regulating glioma cells. In addition, it is necessary to confirm the different effects of CAPON-L overexpression on glioma cell growth in patient-derived cell lines and an animal model.
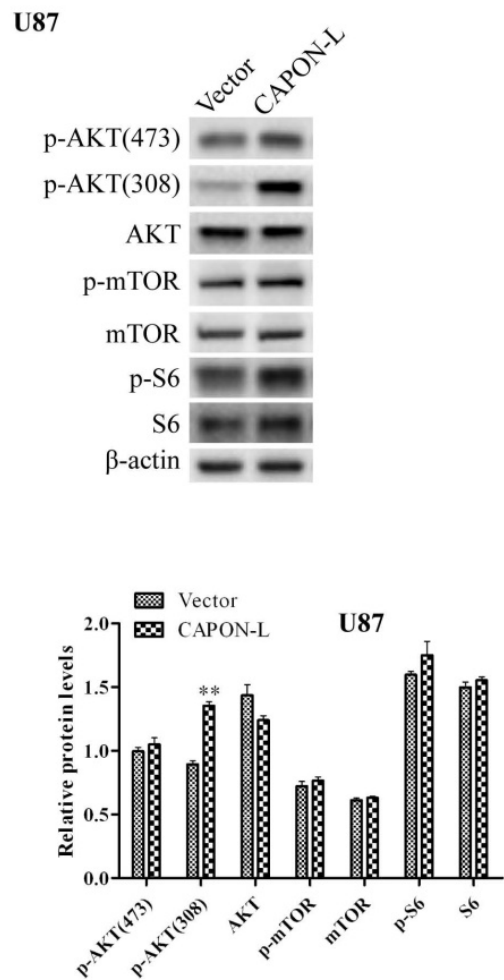

$\mathrm{D}$

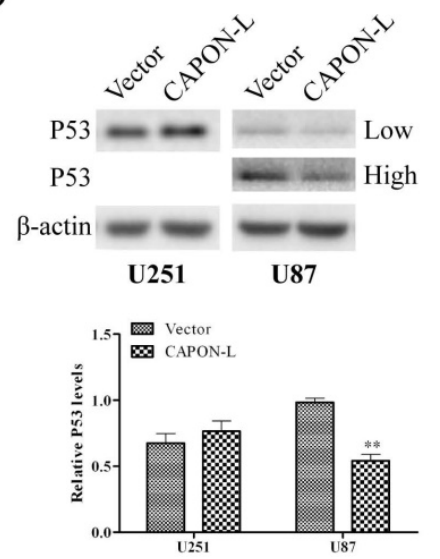

Figure 5. Effects of CAPON-L overexpression on the AKT, mTOR and P53 signaling transduction. Western blot was used to detect some important molecules in the AKT, mTOR and P53 signaling in CAPON-L-overexpressing U87 cells and U251 cells. (A, B) The overexpression of CAPON-L caused significant increases in the p-AKT (473), p-mTOR, mTOR, p-S6 and S6 levels in U251 cells, while overexpressing CAPON-L showed a significant increase in the p-AKT (308) level and no significant effects on the other signaling molecules in U87 cells. (C) Transfecting constitutively active AKT (myr-AKT) partially reversed the decreased phosphorylation of AKT and S6 caused by the CAPON-L overexpression in U251 cells. (D) The overexpression of CAPON-L led to an increase in the muted P53 level in U251 cells, whereas it significantly decreased the wild-type P53 level in U87 cells. Low: low exposure; High: high exposure. $(* P<0.05$; $* * P<0.01$; $* * * P<$ $0.001)$. 
A

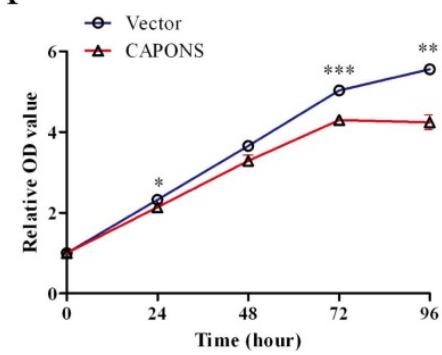

$\mathrm{D}$

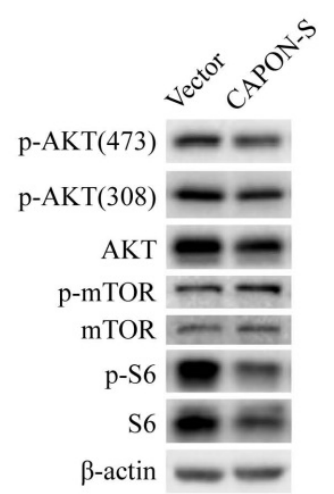

B

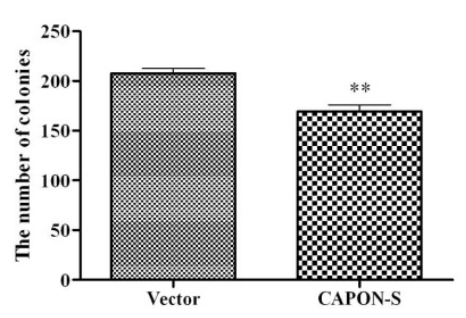

E

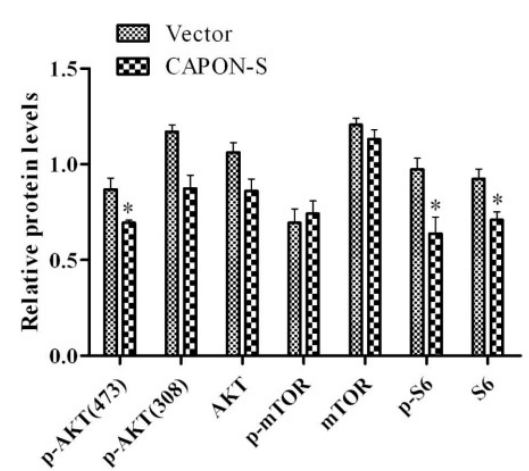

Figure 6. Effects of CAPON-S overexpression on cell proliferation, cell cycle progression and AKT/mTOR signaling transduction in U251 cells. (A) CCK8 assay showed that the overexpression of CAPON-S significantly decreased the cell viability at $24 \mathrm{~h}, 72 \mathrm{~h}$ and $96 \mathrm{~h}$. (B) Colony formation assay revealed a significant decrease in the number of colonies in the CAPON-S group. (C) Flow cytometry showed that the overexpression of CAPON-S resulted in more cells in the Gl/G0 phase and less cells in the $\mathrm{S}$ phase compared with the Vector group. (D, E) Western blot analysis showed that overexpressing CAPON-S caused significant decreases in the p-AKT (473), p-S6 and S6 levels in U251 cells. (* $P<0.05 ; * * P<0.01 ; * * * P<0.001)$.

\section{Conclusions}

Combined with our previous work [20] and the present findings, both CAPON-L and CAPON-S play an inhibitory role in glioma cell proliferation through the AKT/mTOR signaling pathway. It should be noted that the overexpression of CAPON-L may have a promoting effect on glioma cell proliferation in human cell lines bearing wild-type P53. Our work lays a solid foundation for the targeting of CAPON in the treatment of glioma.

\section{Supplementary Material}

Supplementary figures.

http://www.medsci.org/v16p0614s1.pdf

\section{Abbreviations}

NO: nitric oxide; NOS1: NO synthase 1; CAPON: Carboxy-terminal PDZ ligand of NOS1; PTB: phosphotyrosine-binding; PDZ: PSD-95/discs-large/ ZO-1; YAP: Yes-associated protein; GFP: green fluorescent protein; OD: optical density; CDK: cyclindependent kinase; mTOR: mammalian target of rapamycin; S6: ribosomal protein $\mathrm{S6}$.

\section{Acknowledgments}

This work was supported by the National Natural Science Foundation of China (No. 31400930;
81802490), the Natural Science Foundation of Jiangsu Province (No. BK20140217), the Key Research \& Development Plan of Jiangsu Province (No. BE20166 46; BE2017636), and the China Postdoctoral Science Foundation (No. 2016T90505). S. G. was supported by the Jiangsu Provincial Medical Youth Talent (No. QNRC2016787) and the Jiangsu Provincial Qing Lan Project. The myr-AKT plasmid was a gift from Dr. Mingshan Niu (Xuzhou Medical University, Jiangsu, China).

\section{Competing Interests}

The authors have declared that no competing interest exists.

\section{References}

1. Wen PY, Kesari S. Malignant gliomas in adults. The New England journal of medicine. 2008; 359: 492-507.

2. Ohgaki H, Kleihues P. Population-based studies on incidence, survival rates, and genetic alterations in astrocytic and oligodendroglial gliomas. Journal of neuropathology and experimental neurology. 2005; 64: 479-89.

3. Stupp R, Hegi ME, Mason WP, van den Bent MJ, Taphoorn MJB, Janzer RC, et al. Effects of radiotherapy with concomitant and adjuvant temozolomide versus radiotherapy alone on survival in glioblastoma in a randomised phase III study: 5-year analysis of the EORTC-NCIC trial. Lancet Oncol. 2009; 10: 459-66.

4. Castro MG, Candolfi M, Kroeger KM, King GD, Curtin JF, Yagiz K, et al. Gene Therapy and Targeted Toxins for Glioma. Curr Gene Ther. 2011; 11: 155-80.

5. Jaffrey SR, Snowman AM, Eliasson MJ, Cohen NA, Snyder SH. CAPON: a protein associated with neuronal nitric oxide synthase that regulates its interactions with PSD95. Neuron. 1998; 20: 115-24.

6. Arking DE, Pfeufer A, Post W, Kao WH, Newton-Cheh C, Ikeda M, et al. A common genetic variant in the NOS1 regulator NOS1AP modulates cardiac repolarization. Nat Genet. 2006; 38: 644-51. 
7. Segalat L, Grisoni K, Archer J, Vargas C, Bertrand A, Anderson JE. CAPON expression in skeletal muscle is regulated by position, repair, NOS activity, and dystrophy. Exp Cell Res. 2005; 302: 170-9.

8. Hu C, Wang C, Zhang R, Ng MC, Bao Y, Wang C, et al. Association of genetic variants of NOS1AP with type 2 diabetes in a Chinese population. Diabetologia. 2010; 53: 290-8.

9. $\mathrm{Xu} \mathrm{B}$, Wratten N, Charych EI, Buyske S, Firestein BL, Brzustowicz LM. Increased expression in dorsolateral prefrontal cortex of CAPON in schizophrenia and bipolar disorder. PLoS Med. 2005; 2: e263.

10. Wang J, Jin L, Zhu Y, Zhou X, Yu R, Gao S. Research progress in NOS1AP in neurological and psychiatric diseases. Brain research bulletin. 2016; 125: 99-105.

11. Lala PK, Chakraborty C. Role of nitric oxide in carcinogenesis and tumour progression. The Lancet Oncology. 2001; 2: 149-56.

12. Fang M, Jaffrey SR, Sawa A, Ye K, Luo X, Snyder SH. Dexras1: a G protein specifically coupled to neuronal nitric oxide synthase via CAPON. Neuron. 2000; 28 : 183-93.

13. Ryan MB, Corcoran RB. Therapeutic strategies to target RAS-mutant cancers. Nature reviews Clinical oncology. 2018.

14. Clattenburg L, Wigerius M, Qi J, Rainey JK, Rourke JL, Muruganandan S, et al. NOS1AP Functionally Associates with YAP To Regulate Hippo Signaling. Mol Cell Biol. 2015; 35: 2265-77.

15. Humbert PO, Grzeschik NA, Brumby AM, Galea R, Elsum I, Richardson HE. Control of tumourigenesis by the Scribble/Dlg/Lgl polarity module. Oncogene. 2008; 27: 6888-907.

16. Zhang K, Qi HX, Hu ZM, Chang YN, Shi ZM, Han XH, et al. YAP and TAZ Take Center Stage in Cancer. Biochemistry. 2015; 54: 6555-66.

17. Alzahrani F, Clattenburg L, Muruganandan S, Bullock M, MacIsaac K, Wigerius $\mathrm{M}$, et al. The Hippo component YAP localizes in the nucleus of human papilloma virus positive oropharyngeal squamous cell carcinoma. Journal of otolaryngology - head \& neck surgery = Le Journal d'oto-rhino-laryngologie et de chirurgie cervico-faciale. 2017; 46: 15.

18. Anastas JN, Biechele TL, Robitaille M, Muster J, Allison KH, Angers S, et al. A protein complex of SCRIB, NOS1AP and VANGL1 regulates cell polarity and migration, and is associated with breast cancer progression. Oncogene. 2012; 31: 3696-708.

19. Shen Y, Liu H, Gu S, Wei Z, Liu H. The role of Capon in multiple myeloma. Tumour biology : the journal of the International Society for Oncodevelopmental Biology and Medicine. 2017; 39: 1010428317713674.

20. Gao S, Wang J, Zhang T, Liu G, Jin L, Ji D, et al. Low Expression of CAPON in Glioma Contributes to Cell Proliferation via the Akt Signaling Pathway. International journal of molecular sciences. 2016; 17.

21. Gao S, Zhang T, Jin L, Liang D, Fan G, Song Y, et al. CAPON Is a Critical Protein in Synaptic Molecular Networks in the Prefrontal Cortex of Mood Disorder Patients and Contributes to Depression-Like Behavior in a Mouse Model. Cerebral cortex. 2018

22. Gao S, Jin L, Liu G, Wang P, Sun Z, Cao Y, et al. Overexpression of RASD1 inhibits glioma cell migration/invasion and inactivates the AKT/mTOR signaling pathway. Scientific reports. 2017; 7: 3202.

23. Shen A, Gao S, Ben Z, Wang H, Jia J, Tao T, et al. Identification and potential role of PSD-95 in Schwann cells. Neurological sciences : official journal of the Italian Neurological Society and of the Italian Society of Clinical Neurophysiology. 2008; 29: 321-30.

24. Zhang T, Ji D, Wang P, Liang D, Jin L, Shi H, et al. The atypical protein kinase RIOK3 contributes to glioma cell proliferation/survival, migration/invasion and the AKT/mTOR signaling pathway. Cancer letters. 2018; 415: 151-63.

25. Abraham AG, O'Neill E. PI3K/Akt-mediated regulation of p53 in cancer. Biochemical Society transactions. 2014; 42: 798-803.

26. Nakada M, Kita D, Watanabe T, Hayashi Y, Teng L, Pyko IV, et al. Aberrant signaling pathways in glioma. Cancers. 2011; 3: 3242-78.

27. Hannan KM, Sanij E, Hein N, Hannan RD, Pearson RB. Signaling to the ribosome in cancer--It is more than just mTORC1. IUBMB life. 2011; 63: 79-85. 